\title{
MICROFOSSILS IN THE ORDOVICIAN ERRATIC BOULDERS FROM SOUTHWESTERN FINLAND
}

\author{
JAAK NÕLVAK, TÕNU MEIDLA and ANNELI UUTELA
}

NÕLVAK, JAAK; MEIDLA, TÕNU and UUTELA, ANNELI 1995. Microfossils in the Ordovician erratic boulders from South-western Finland. Bull. Geol. Soc. Finland 67, Part II, 3-26.

Chitinozoans, ostracods and acritarchs found in four glacially transported limestone boulders from the south-western coast of Finland have been studied in order to test the usefulness of these microfossil groups in age determinations. Also rare specimens of conodonts, inarticulated brachiopods and foraminifers were found.

Baltic limestone (or Östersjö limestone) was the most problematic, because only fossils with calcitic or phosphatic shells are preserved.

It is concluded that the boulders identified correlate with the Uhaku and Rakvere stages of the Middle Ordovician.

Key words: microfossils, sedimentary rocks, limestone, erratics, chitinozoans, ostracods, acritarchs, Ordovician, Southwestern Finland.

Jaak Nõlvak, Estonian Academy of Sciences, Institute of Geology, Estonia Avenue 7, EE-0100 Tallinn, Estonia;

Tõnu Meidla, Institute of Geology, Tartu University, Vanemuise 46, EE-2400 Tartu, Estonia;

Anneli Uutela, Finnish Museum of Natural History, Geological Museum, Paleontology Department, P.O. Box 11, FIN-00014 University of Helsinki, Finland

\section{INTRODUCTION}

The lower Palaeozoic sedimentary rocks occur in the basin of the Bothnian Sea (Winterhalter et al. 1981) and numerous glacially transported boulders are distributed along the south-western coast of
Finland (Uutela 1989). Since until now the borehole data from the Bothnian Sea is scarce, the study of erratics provide the only suitable method for determining the extent of the early Palaeozoic northern Baltic Basin. Because of the small size of the boulders acritarchs have been valuable for 
dating these boulders (Martinsson 1956, Eisenack 1965, Tynni 1975, Uutela 1989).

The aim of the study was to test the reliability of planktonic acritarchs in dating boulders. Except for the Rapla borehole in Estonia (Uutela \& Tynni 1991) acritarchs have not yet been widely studied in the Baltoscandian Ordovician sections, especially by using filtering method in preparation, which makes comparison between different areas difficult. However, chitinozoans are the most widely studied (Grahn 1980, 1981a,b, 1982, 1984, Nõlvak \& Grahn 1993) and more than 20 sections have been recently investigated in the East Baltic region (Nõlvak, unpublished material), while ostracods are studied in Estonia by Sarv (1959) and Meidla (1986, 1993, in press), in Latvia by Gailite (1982) and in Lithuania by Sidaravičiene (1992).

\section{MATERIAL AND METHODS}

The material studied comprises four limestone erratic boulders from Pyhäranta, Pyhämaa, Kalanti and Kustavi, from the south-western coast of Finland (Fig. 1). A large amount of material was needed in order to date microfossiliferous boulders.

Chitinozoans have been prepared with a weak acetic acid using $0.5 \mathrm{~kg}$ sample size. Ostracods have been prepared by "sodium acetate method" (Sohn 1961), where the amount of a sample was $0.3-1 \mathrm{~kg}$. In the maceration process of acritarchs $\mathrm{HF}$ and $\mathrm{HCl}$ were used before filtering the residue (Vidal 1988) and about $20 \mathrm{~g}$ of limestone were used. Responsibilities for authorship are as follows: Jaak Nõlvak chitinozoans and lithological description of boulders, Tõnu Meidla ostracods and Anneli Uutela acritarchs.

\section{DESCRIPTION OF SEDIMENTARY ROCKS}

Macroscopically the boulders investigated are similar to those Ordovician limestones in North Estonia, that are considered to belong to the North
Estonian Confacies Belt (see Jaanusson 1976, Figs $6,7)$. Some lithological affinities link studied blocks to the cryptocrystalline limestone that characterizes either the Rakvere and Nabala stages (upper Viru - lower Harju series) or the "building limestone" from the Lasnamägi - Uhaku stages (lower Viru series). Within these limestones relatively hard (i.e. less argillaceous) types are present. Macroscopically finely dispersed haematite can be observed in Kalanti and Kustavi boulders, although this feature is not common in the North Estonian outcrop area, where illite and in places finely dispersed quartz usually comprise the main terrigenous component of limestones.

A distinctly fractured subangular boulder from Pyhäranta (Pl. I:1, 2) is a grey calcarenite (see Jaanusson 1972), dolomitic limestone characterized by $26 \%$ of skeletal grains (bioclasts) (Fig. 2). Limestone layers are more than $4 \mathrm{~cm}$ thick and a weak phosphatic discontinuity surface is recognized within the boulder (P1. I:1). Concentrations of goethite and also small amounts of pyrite occur in the ca. $2 \mathrm{~cm}$ deep borings. The content of trilobite and echinoderm, as well as indeterminable fragments is over $20 \%$. The matrix crystal size is up to $0.01 \mathrm{~mm}$.

The Pyhämaa boulder (Pl. I:3, 4) is heterogenous, containing yellow-grey, finely nodular (2-3 cm in diameter) secondary dolomite with darker yellowish-grey argillaceous lamellae $0.5-1 \mathrm{~cm}$ thick (calcilutite; see Jaanusson 1972). Based on the chemical and X-ray analyses the dolomite content (i.e. the extent of the dolomitization) in the rock is between $60-85 \%$. However, some parts show only $59.9 \%$ of dolomite and therefore the "typical" content of the Pyhämaa boulder is not showed in Fig. 3 .

Mostly unidentifiable skeletal grains (up to $4 \%$, suggesting primary calcilutite) are affected by the dolomitization (Pl. I, Fig. 7). In thin section idiomorphic dolomite crystals (up to $0.1 \mathrm{~mm}$, mostly less than $0.05 \mathrm{~mm}$ ) can be identified. Finely nodular surface structure is only slightly rounded and cavernous (ca. $1 \mathrm{~cm}$ deep). The Pyhämaa boulder is in very similar to the boulders in Figs 4 D, E in Uutela (1989). 


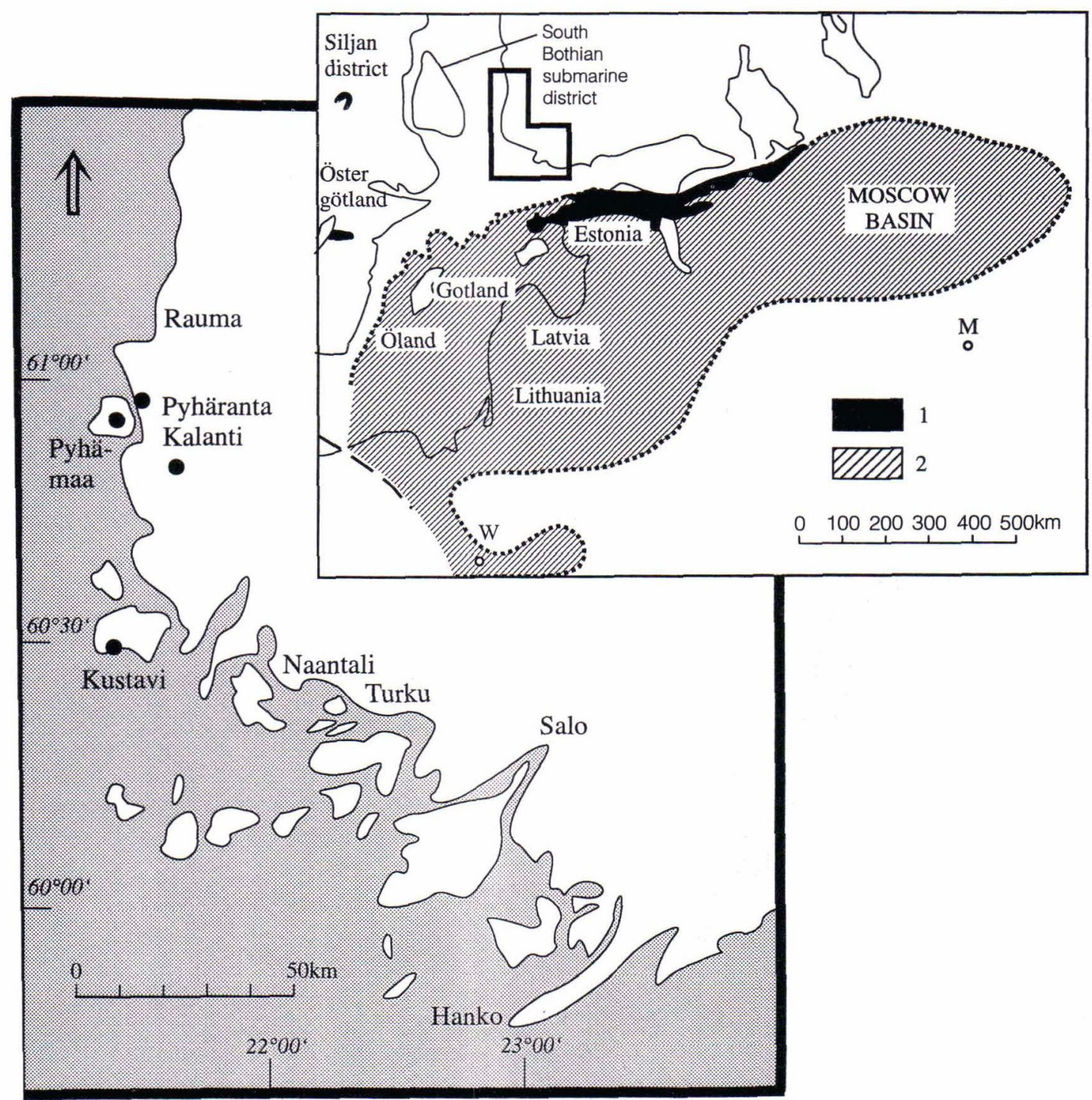

Fig. 1. Location map. The location of the glacially transported boulders investigated in this study. Outcrops areas of Ordovician deposits in the East Baltic and eastern Sweden (black) and the extent of subsurface and submarine Ordovician on the East European Platform (diagonal shading); $W=$ Warzawa, $M=$ Moscow (after Jaanusson 1976).

The Kalanti boulder (Pl. I:5, 6) represents a reddish to brownish calcilutite, dolomitic limestone that contains $2 \%$ bioclastic material. The primary orientation of bedding planes is not visible, although the original bed thickness has been more than $8 \mathrm{~cm}$. The unevenly distributed reddish tint is caused by haematite. The micritized skeletal grains are unidentifiable. The inclusions of sparry calcite, sometimes with haematite supplement, can be recognized on the polished limestone surface. The conchoidal fracture is characteristic, which suggests the matrix chrystal- 


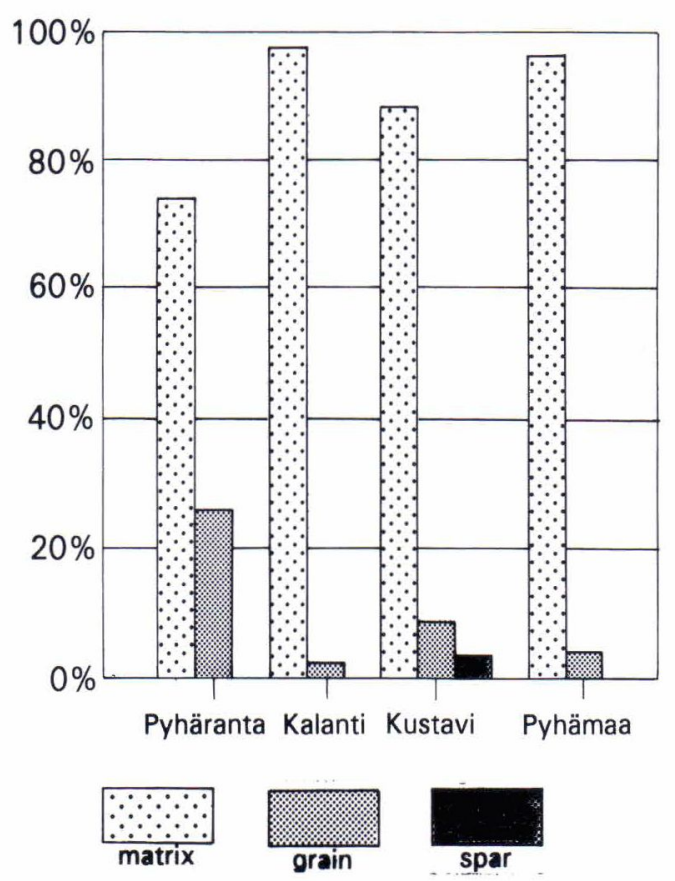

Fig. 2. Grain composition of the Pyhäranta, Pyhämaa, Kalanti and Kustavi boulders.

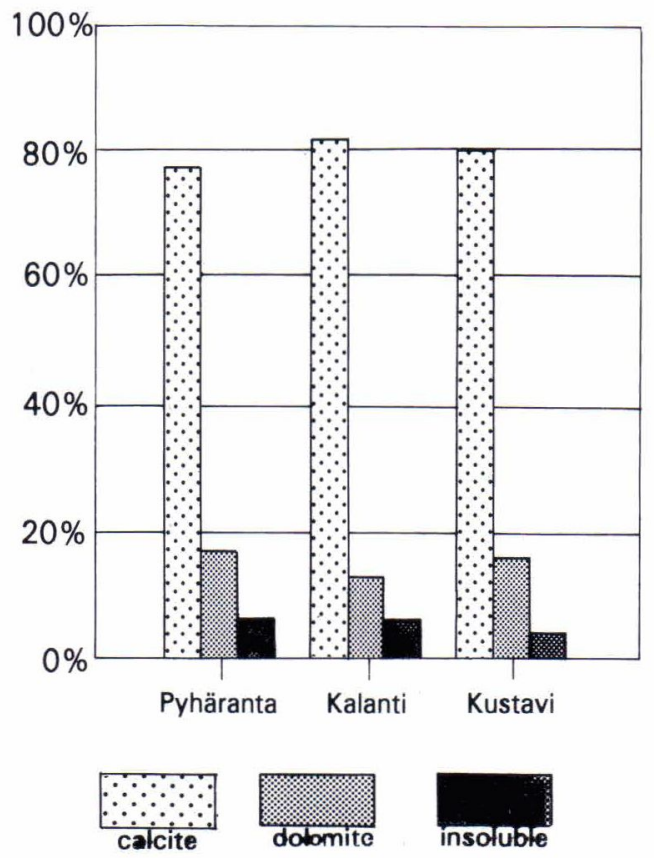

Fig. 3. Main constituents of the Pyhäranta, Kalanti and Kustavi boulders. size is less than $0.005 \mathrm{~mm}$. The boulder is very similar in colour and structure to the boulder described by Uutela (1989, Fig. 4F).

In the Kalanti boulder a distinct weathering zonation is present and results from the differences in iron mineralogy. The primary reddishbrown colour of the rock grades into dark grey in the upper part of the block (about $1.5 \mathrm{~cm}$ from the upper surface). The outermost weathered goethitebearing crust, which is ca. $9 \mathrm{~mm}$ thick, evenly laminated and distinctly tripartite consists of: 1) an inner layer that has a weak goethite impregnation, 2) an outer strongly weathered part that consists of a goethite-rich part and 3) almost goethite-free, partly laminated layers.

The Kustavi boulder may also be classified as calcilutite, dolomitic limestone (Pl. I:5, 6). The grain component reaches up to $12 \%$, comprising $3.4 \%$ sparry calcite and $8.6 \%$ skeletal grains (Fig. 3 ). Among the skeletal grains brachiopods comprise over $20 \%$, echinoderms $15 \%$ and other groups less than $10 \%$. A total of $30 \%$ of grains were impossible to identify. The original bedding thickness has been over $5 \mathrm{~cm}$.

The surface of the boulder is covered by a distinct 6-7 $\mathrm{mm}$ thick weathered zone, with a continuous transition to the unweathered reddishbrown limestone, coloured by finely dispersed haematite. The Kustavi boulder is similar to the block in Uutela (1989, Fig. 4F).

The terminology of the Estonian regional stages is used here for the biochronostratigraphical correlation (Fig. 4).

\section{CHITINOZOANS}

Chitinozoans are extinct microscopic animals whose systematic position is still unknown. Their classification is based on the shape and ornamentation of the organic-walled tests. Chitinozoans were exclusively marine, and most probably planktonic. They evolved rapidly, a fact that makes them valuable guide fossils for stratigraphic correlation. More than 130 species are known from the Ordovician and also broken 


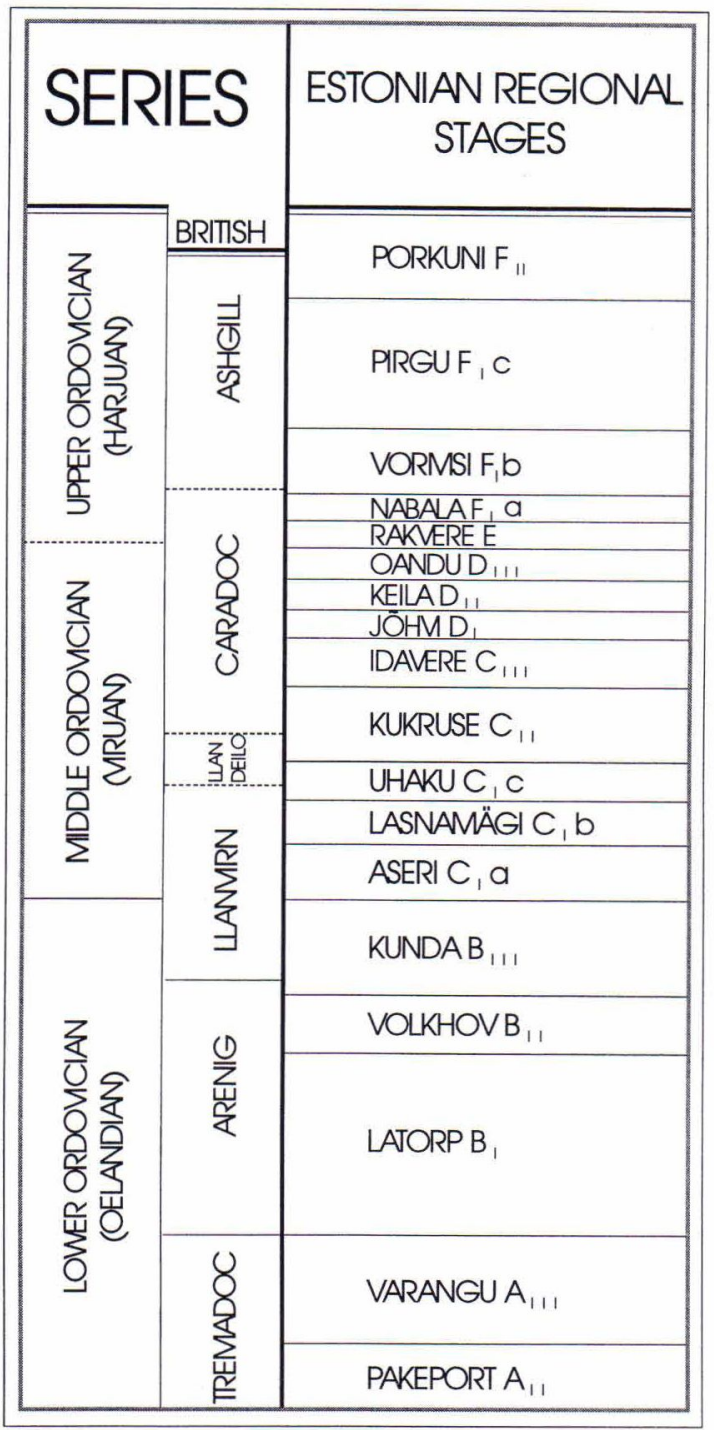

Fig. 4. Correlation of the Ordovician Estonian regional stages with the British and Baltoscandian Series (Nõlvak \& Grahn 1993).

specimens can be identified. Chitinozoans are well preserved in the material and occur only in Pyhäranta and Pyhämaa boulders.

A total of eleven chitinozoan species were preserved in the Pyhäranta boulder, most of which are with a long stratigraphic range. The coexistence of Conochitina clavaherculi Eisenack 1959 (Pl. II:14), Desmochitina complanata Eisenack 1932
(P1. II:12) and Cyathochitina kuckersiana (Eisenack 1934) (Pl. II: 17) can be correlated to the early Uhaku age in the beginning of the Middle Ordovician, according to their distribution in Estonian biochronology (Table 1).

In the Pyhämaa boulder five chitinozoan species were found. The most important of these is Cyathochitina angusta Nõlvak and Grahn 1993, which together with Belonechitina robusta (Eisenack 1959) (Pl. II:4) determine the upper part of Middle Ordovician, i.e. the Rakvere Stage (Table 2).

\section{OSTRACODS}

Small crustaceans, ostracods are valuable guide fossils in shallow sea deposits. Their classification is based on the symmetry/asymmetry of the calcified valve (carapace) and on several external features (e.g. shape, dimorphic structures, ornamentation) and internal features (e.g. free margin structures, adductor muscle scar, hinge). One of the richest Ordovician ostracod faunas of the world has been described from Baltoscandia, and stratigraphic distribution of most taxa is well known in the East Baltic, Poland and Scandinavia. Benthic mode of life makes ostracods sensitive to environmental changes and the main carbonate lithofacies of the East Baltic are characterized by specific ostracod associations, which evolved rapidly during the Ordovician.

In the present material ostracods are rather well preserved. Apparently the difficulties in disintergation of hard limestone varieties and/or possible rapid sedimentation, mean that the number of specimens found in the blocks is small. The samples contain poorly preserved specimens, in addition to fragments and very young instars, which cannot be identified with a certainty. However, a mostly sufficient number of species were found for correlation purposes. Baltic limestone have generally been dated by using ostracods.

The Pyhäranta boulder was poor in ostracods, although one specimen, propably Baltonotella sp. (Pl. V:2a, b; Table 1). Two very similar ostracod 


\begin{tabular}{|c|c|c|c|c|c|c|c|c|c|c|}
\hline Pyhäranta, Rihtniemi 55/AU/85 & $B_{l u}$ & $C_{I} a$ & $\bar{C} b \bar{C}$ & $C_{i} c$ & $C_{u}$ & $C_{m}$ & $D_{t}$ & \begin{tabular}{|l|l}
$D_{u}$ & $D$ \\
\end{tabular} & $\overline{D_{u t}}$ & No \\
\hline CHITINOZOANS & & & (i) & & & & & & & \\
\hline Belonechitina micracantha & & & $=$ & & & & & - & $\rightarrow$ & 14 \\
\hline Cyathochitina calix & $\leftarrow$ & & 二 & & & & - & & & 42 \\
\hline C. kuckersiana & & & & & & & & & $\rightarrow$ & 20 \\
\hline Conochitina clavaherculi & & & E & - & & & & & & 16 \\
\hline C. primitiva & & & $=$ & & & & & - & & 94 \\
\hline Desmochitina amphorea & & & E & & & & & - & & 3 \\
\hline D. complinata & & & & & & & & & $\rightarrow$ & 10 \\
\hline D. elongata & $\leftarrow$ & & E & & E & & & & & 14 \\
\hline D. erinacea & $\leftarrow$ & & B & & E & & & & & 11 \\
\hline D. ovulum & & & E & E & E & & & & & 18 \\
\hline Rhabdochitina gracilis & 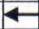 & & $\Longrightarrow$ & & & & & 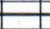 & $\rightarrow$ & 10 \\
\hline OSTRACODES & & & m & & & & & & & \\
\hline Baltonotella? sp. & 4. & $\cdots$ & $\cdots$ & . & $\cdots$ & $\cdots$ & $\cdots$ & $\cdots$ & $\rightarrow$ & 1 \\
\hline ACRITARCHS & & & - & & & & & & & \\
\hline Baltisphaeridium bystrentos & & & E & & - & & & - & $\rightarrow$ & 1 \\
\hline B. eisenackianum & & & E & & & & & 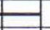 & & 1 \\
\hline B.hirsutoides & $\leftarrow$ & & $\square$ & & - & & & - & $\rightarrow$ & 1 \\
\hline B.longispinosum & $\leftarrow$ & - & E & - & E & & - & 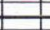 & $\rightarrow$ & 3 \\
\hline B. microspinosum & 4 & E & $\square$ & 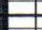 & E & & - & - & $\rightarrow$ & 4 \\
\hline B. mochtiense & & - & E & E & & & & & & 1 \\
\hline B.multiechinatum & $\leftarrow$ & & E & 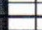 & - & - & & & & 10 \\
\hline B.nanninum & & & $\square$ & & 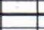 & & & & $\rightarrow$ & 2 \\
\hline B.pseudocalicispinum & $\leftarrow$ & & $\Longrightarrow$ & E & E & & & 二 & $\rightarrow$ & 1 \\
\hline B. pustulatum & & & E & - & 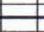 & & 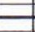 & 二 & $\rightarrow$ & 1 \\
\hline Buedingiisphaeridium balticum & - & & E & E & E & & - & 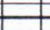 & $\rightarrow$ & 3 \\
\hline Chlamydosphaeridia sp. & & & E & - & E & 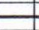 & - & - & & 1 \\
\hline Comasphaeridium filosum & $\leftarrow$ & - & 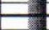 & E & E & & E & 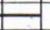 & & 1 \\
\hline Cymatiosphaera pavimenta & $\leftarrow$ & & ב & & & & & & $\rightarrow$ & 1 \\
\hline Filisphaeridium eatonense & & & 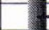 & 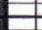 & - & & & Z & $\rightarrow$ & $I$ \\
\hline F. henryi & 4 & & $\square$ & E & - & & - & E & $\rightarrow$ & 33 \\
\hline F. lasnamaegiense & & & E & 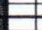 & 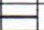 & & & - & & 13 \\
\hline Gorgonisphaeridium antiquum & $\leftarrow$ & & $\theta$ & E & 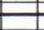 & & - & 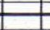 & $\rightarrow$ & 2 \\
\hline Kundasphaera lacunosa & $\leftarrow$ & - & $E$ & E & E & & $=$ & 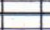 & $\rightarrow$ & 1 \\
\hline Leiovalia similis & $\leftarrow$ & - & $\Longrightarrow$ & E & E & & - & 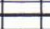 & $\rightarrow$ & 1 \\
\hline Lophosphaeridium citrinipeltatum & $\leftarrow$ & & $E$ & E & E & & E & 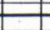 & $\rightarrow$ & 1 \\
\hline L.papillatum & $\leftarrow$ & & $\Longrightarrow$ & E & E & & & + & $\rightarrow$ & 3 \\
\hline L. pilosum & $\leftarrow$ & & E & E & $\square$ & & & & $\rightarrow$ & 1 \\
\hline Micrhystridium inaffectum & - & 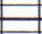 & 世 & 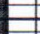 & - & & & E & $\rightarrow$ & 1 \\
\hline M. nannacanthum & $\leftarrow$ & & $\square$ & E & $E$ & & & 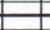 & $\rightarrow$ & 49 \\
\hline M. aff. M. parinconspicuum & $\leftarrow$ & - & $\Longrightarrow$ & & E & & - & 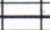 & $\rightarrow$ & 12 \\
\hline M. shinetonensis & & & - & E & 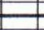 & & - & 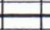 & $\rightarrow$ & 1 \\
\hline M. stellatum & & & E & E & ב- & & Z & E & $\rightarrow$ & 5 \\
\hline M. sp. 2. & $\leftarrow$ & & $\Rightarrow$ & E & - & & & & & 1 \\
\hline Multiplicisphaeridium alloiteauii & 4 & & $\Rightarrow$ & 年 & . & & & 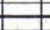 & $\rightarrow$ & 3 \\
\hline M. bipalmatum & & & $\Rightarrow$ & 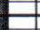 & E & & & 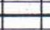 & $\rightarrow$ & 4 \\
\hline M. ferum & & & E & $E$ & & & & & $\rightarrow$ & 2 \\
\hline M. fissile & $\leftarrow$ & & E & & 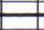 & & & 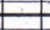 & $\rightarrow$ & 4 \\
\hline M. irregulare & - & & - & & 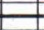 & & & 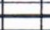 & $\rightarrow$ & 1 \\
\hline M. martae & $\leftarrow$ & & E & & 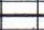 & & & - & $\rightarrow$ & 2 \\
\hline M. parvipinnatum & - & & E & E & 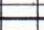 & & - & 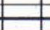 & $\rightarrow$ & 1 \\
\hline M. parvirochesterensis & & & - & - & - & & - & - & $\rightarrow$ & $I$ \\
\hline M. raplaense & - & & $\Longrightarrow$ & - & & & & & $\rightarrow$ & 2 \\
\hline M. raspa & & & 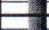 & E & & & & & $\rightarrow$ & 8 \\
\hline M. varipinnosum & $\leftarrow$ & & E & E & & & & - & $\rightarrow$ & 1 \\
\hline Ordovicidium heteromorphicum & & & $\square$ & 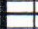 & & & & & $\rightarrow$ & 1 \\
\hline O. nudum & & & E & - & & & & 二 & $\rightarrow$ & 1 \\
\hline Orthossphaeridium densigranosum & & & - & - & & & & & & 1 \\
\hline O. vibrissiferum & & & $\Longrightarrow$ & E & & & & - & $\rightarrow$ & 1 \\
\hline Polyancistrodorus magnispinosus & - & & ב & E & - & & & & & 1 \\
\hline Polygonium pellicidum & - & & C & E & & & & - & $\rightarrow$ & 1 \\
\hline P. polygonale & $\leftarrow$ & & - & 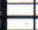 & & & & - & $\rightarrow$ & 2 \\
\hline P. tenuispinosum & $\leftarrow$ & & ב & 日 & & & & & & 1 \\
\hline Raplasphaera undosa & & & Z & E & & & & & $\rightarrow$ & 1 \\
\hline Rhopaliophora pilata & - & - & 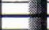 & E & & & & - & $\rightarrow$ & $I$ \\
\hline Timofeevia nodosa & & & ב & & & & & & & 1 \\
\hline Tranvikium pobygonale & & & $\square$ & & & & & & & 1 \\
\hline Veryhachium lairdi & & & Z & 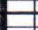 & & & & - & $\rightarrow$ & 1 \\
\hline V. reductum & & & & & & & & - & & 1 \\
\hline V. trispinosum & & & 二 & & & & & Z & $\theta$ & 4 \\
\hline
\end{tabular}

Table 1. The stratigraphical distribution of chitinozoans, ostracods and acritarchs in the Pyhäranta boulder. The stratigraphical distribution of chitinozoans based on works by Laufeld (1967), Eisenack (1976), Grahn (1980, 1981a, 1982, 1984), Männil (1986), Nõlvak \& Grahn (1993); ostracods on Meidla (1986); acritarchs on Kjellström (1971), Uutela \& Tynni (1991). For synonymics of acritarchs see Sarjeant \& Stancliffe (1994).

genera Baltonotella and Brevidorsa characterize most of the Ordovician system (Vannier et al. 1989).

The Pyhämaa boulder can be dated as Rakvere or early Nabala according to co-occurrence of Platybolbina orbiculata (Pl. III:3) and Bolbina major (Pl. III:21). The known Estonian ranges of currently undescribed taxa (Meidla, in press), however, could restrict the dating interval down to late Rakvere (Table 2).

In the Kalanti boulder the ostracod assemblage in general, with the co-occurrence of Bolbina plicata (Pl. III:18), Platybolbina temperata (Pl. III:2) and Seviculina oanduensis (P1. III:1) determines the dating the late Oandu - early Rakvere age (Table 3).

In the Kustavi boulder Leperditella prima ( $\mathrm{Pl}$. III:15) and Rectella romboformis (Pl. IV:4) together with Duplicristata sp. (P1. III:13) determine the Oandu - Rakvere age, preferrably the latter (Table 4).

\section{ACRITARCHS}

Acritarchs are an artificial group of organic-walled cysts (Evitt 1963). Rapid evolution makes them valuable guide fossils of the Neoproterozoic to the Lower Palaeozoic sedimentary deposits. In the North Estonian Rapla section over 300 species are known (Uutela \& Tynni 1991). Many of these 


\begin{tabular}{|c|c|c|c|c|c|c|c|c|c|c|}
\hline Pyhamaa, Lintuluoto 39/AU/85 & \begin{tabular}{|l|l|l}
$C_{n}$ & $C$ \\
\end{tabular} & $C_{m}$ & \begin{tabular}{|l|l|}
$D_{I}$ & $D$ \\
\end{tabular} & \begin{tabular}{|l|l|}
$D_{n}$ & $D_{m}$ \\
\end{tabular} & $E$ & $F_{l} a$ & \begin{tabular}{|l|l|}
$F, b$ & 1 \\
\end{tabular} & \begin{tabular}{|l|l|}
$F_{\mathrm{f}} c$ \\
\end{tabular} & $F_{n}$ & No \\
\hline \multicolumn{11}{|l|}{ CHITINOZOANS } \\
\hline Belonechitina robusta & & & & & B. & & & & & 57 \\
\hline B. wesenbergensis brevis & & & & & E & & & E & & 23 \\
\hline Cyathochitina angusta & & & & & E & - & & & & 2 \\
\hline C. campanulaeformis & & & & & H & & & E & - & 62 \\
\hline Desmochitina minor & & & & & H & 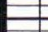 & 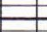 & 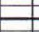 & 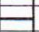 & 21 \\
\hline \multicolumn{11}{|l|}{ OSTRACODES } \\
\hline Bolbina major & & & & -1 & $=$. & $=$ & & & & 3 \\
\hline Hemiaechminoides minusculus & & & & - ? & E & 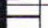 & & & & 1 \\
\hline Longiscula sp. & & & & $\cdots$ &. & & $\cdots$ & $\cdots$ & $\cdots$ & 2 \\
\hline Neckajatia? & & & & $\rightarrow$ & E & & 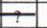 & & & 7 \\
\hline Platybolbina orbiculata & & & & & - & - & -1 & - & -1 & 1 \\
\hline Pseudulrichia inarguta & & & & 一 & - & - & -1 & -1 & & 2 \\
\hline Rectella sp. & & & & $\cdots$ & $\cdots$ & $\because$ & ..... & $\cdots$ & $\cdots$ & 2 \\
\hline Tvaerenella sp. & & & $\cdots$ & . &. & $\cdots$ & $\cdots$ & $\cdots$ & $\cdots$ & 1 \\
\hline Genus B. sp. & & & & & 1 & & & - & - & 1 \\
\hline \multicolumn{11}{|l|}{ ACHRITARCHS: } \\
\hline Axisphaeridium tricolumnelare & & & & & E & & & 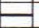 & & 1 \\
\hline Baltisphaeridium aliquigranulatum & & & & & + & & & 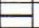 & & 1 \\
\hline B. hirsutoides & 4 & & & & t & & & - & $\rightarrow$ & 6 \\
\hline B. longispinosum & 4 & & & & H & & & - & $\rightarrow$ & 1 \\
\hline B. microspinosum & $\leftarrow$ & & & & H & & & 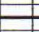 & $\rightarrow$ & 1 \\
\hline B. nanninum & $\leftarrow$ & & & 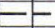 & E & - & -1 & - & - & 1 \\
\hline B. parvigranosum & $\leftarrow$ & & & 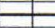 & E & 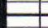 & - & - & & 1 \\
\hline B. pseudocalicispinum & 4 & & & & t & & & & $\rightarrow$ & 1 \\
\hline Buedingiisphaeridium balticum & 4 & & & & H & & - & 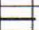 & & 1 \\
\hline B. brevispinosum & $\leftarrow$ & & & & E & & & 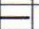 & & 1 \\
\hline Comasphaeridium sp. 1 & $\leftarrow$ & & & & $t$ & & & 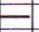 & & 1 \\
\hline Cymatiosphaera crispa & & & & & tE & & & & & 1 \\
\hline C. pavimenta & $\leftarrow$ & & & & $\theta$ & & & & & 2 \\
\hline Dilatisphaera tubulifera & 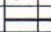 & & & & H & & & — & & 1 \\
\hline Dorsennidium oligospinoides & & & & & He & & & 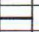 & & 1 \\
\hline Filisphaeridium eatonense & 4 & & & & 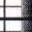 & & & & $\rightarrow$ & 1 \\
\hline F. henryi & $\leftarrow$ & & & & +2 & & & 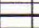 & $\rightarrow$ & 31 \\
\hline F parvulum & & & & & H & & & - & $\Rightarrow$ & 2 \\
\hline Gorgonisphaeridium spiralispinosum & 4 & & & & + & & & 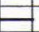 & & 1 \\
\hline Helosphaeridium varispinosum & 4 & & & 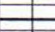 & He & & & 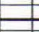 & & 1 \\
\hline Kundasphaera lacunosa & 4 & & & & E & & & & & 2 \\
\hline Leiofusa granulacutis & $\leftarrow$ & & & & + & & & - & & 1 \\
\hline Leiovalia similis & $\leftarrow$ & & & & 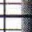 & & & & $\rightarrow$ & 3 \\
\hline Lophosphaeridium aculeatum & $\leftarrow$ & & & & + & & & & 且 & 1 \\
\hline L. citrinipeltatum & $\leftarrow$ & & & & H & & & & $\rightarrow$ & 3 \\
\hline L. papillatum & $\leftarrow$ & & & & E. & & & E & & 2 \\
\hline L. pilosum & 4 & & & & H & 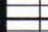 & E & E & & $I$ \\
\hline Micrhystridium exiguum & $\leftarrow$ & & & & + & & & & & 1 \\
\hline M.inaffectum & $\leftarrow$ & & & & H & & & 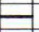 & & 3 \\
\hline M. nannacanthum & $\leftarrow$ & & & & tris & & & 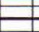 & $\rightarrow$ & 60 \\
\hline M. aff. M. parinconspicuum & $\leftarrow$ & & & & H & & & 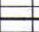 & & 33 \\
\hline M. shinetonensis & 4 & & & & 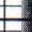 & & & - & & 1 \\
\hline M. stellatum & $\leftarrow$ & & & & 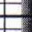 & & & & $\rightarrow$ & 7 \\
\hline M. salopiense & & & & & + & - & & - & $\rightarrow$ & 1 \\
\hline M. sp. 1 & & & & 4 & +1 & - & & & & 10 \\
\hline Multiplicisphaeridium alloiteauii & $\leftarrow$ & & & & E & & & 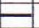 & & 2 \\
\hline M. bifurcatum & $\leftarrow$ & & & & F & & & - & $\rightarrow$ & 2 \\
\hline M. bipalmatum & $\leftarrow$ & & & & E & & & 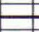 & $\rightarrow$ & 1 \\
\hline M. fisherii & & - & & & t & & 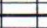 & E & & 1 \\
\hline M. fissile & $\leftarrow$ & & & & t & & 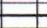 & 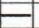 & & 2 \\
\hline M. forquiferum & & & & & 4 & & - & 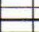 & -1 & 1 \\
\hline M. irrequlare & $\leftarrow$ & & & & E & & & & $\rightarrow$ & 10 \\
\hline M. martae & $\leftarrow$ & & & & E & & & Z & $\rightarrow$ & 1 \\
\hline M. varipinnosum & $\leftarrow$ & & & & E & & & & & 1 \\
\hline Nanocyclopia? sp. & $\leftarrow$ & & & & - & & & E & & 1 \\
\hline Ordovicidium nudum & $\leftarrow$ & & & & E & & & & & $I$ \\
\hline Polyancistrodorus columbariferus & & & & & + & 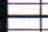 & - & & & 1 \\
\hline Polygonium acuminosum & & & & & E & & & & & 1 \\
\hline P. latispinosum & $\leftarrow$ & & & & F & - & + & & & 1 \\
\hline P. pelliculum & $\leftarrow$ & & & & + & - & & & $\rightarrow$ & 2 \\
\hline P. polygonale & 4 & & & & H & - & - & & $\rightarrow$ & 2 \\
\hline Revinotesta granulosa & & & & & H & & & & & 1 \\
\hline Tunisphaeridium spinosissimum & & & & & E & $=$ & & & & 1 \\
\hline Veryhachium laindi & $\leftarrow$ & & & & $\theta$ & & & & & 2 \\
\hline
\end{tabular}

Table 2. The stratigraphical distribution of chitinozoans, ostracods and acritarchs in the Pyhämaa boulder. The stratigraphical distribution of chitinozoans base on works by Eisenack (1972), Grahn (1980, 1982), Nõlvak \& Grahn (1993) and Nôlvak (unpublished material); ostracods on Krause (1892), Sarv (1959), Neckaja (1966), Meidla (1986), Sidaravi ciene (1992) and Meidla (unpublished material); acritarchs on Eisenack (1965), Schulz (1967), Uutela \& Tynni (1991). For synonymics of acritarchs see Sarjeant \& Stancliffe (1994).

species have a long stratigraphic range, which makes them less suitable for correlation compared to chitinozoans. As well as chitinozoans, also poorly preserved acritarchs are possible to identify, although in the Baltoscandia they are well preserved compared to those in central or southern Europe.

In the Pyhäranta boulder small acritarchs are rather well preserved. Most of a total of 55 species are with a long stratigraphic range (from Lower Ordovician to Lower Silurian). Baltisphaeridium mochtiense Górka 1969 (P1. VI:4) and Tranvikium polygonale Tynni 1982 (Pl. VI:5) disappear in the end of the Lasnamägi Stage. Filisphaeridium eatonense (Pl. VI:6) and Veryhachium reductum (Deunff 1959) Dovnie \& Sarjeant 1965 (Pl. VI:7) appear in the Uhaku Stage. Timofevia nodosa Uutela \& Tynni 1991 (Pl. VI:8) is restricted to the Lasnamägi Stage. The assemblage therefore indicates the transition of the Lasnamägi and Uhaku stages (Table 1).

In the Pyhämaa boulder a total of 54 well preserved, small species with a long-life range dominate. Of these Revinotesta granulosa Uutela \& Tynni 1991 (Pl. VI:1) is restricted to the Rakvere Stage, when Tunisphaeridium spinosissimum Uutela \& Tynni 1991 (Pl. VI:2) and Multiplicisphaeridium forquiferum (Cramer \& Díez 1972) appear and Cymatiosphaera crispa Uutela \& Tynni 1991 (Pl. VI:3) disappears. These species give an age of Rakvere Stage for the boulder (Table 2). 


\begin{tabular}{|c|c|c|c|c|c|c|c|c|c|}
\hline Kalanti, Petes, 63/AU/85 & $D_{r}$ & $D_{n}$ & $D_{m}$ & $\vec{E}$ & $F_{t} a$ & $F b$ & $F_{i} c$ & $F_{n}$ & No. \\
\hline OSTRACODES & & & & & & & & & \\
\hline Bolbina major & & - & E & & $F$ & & & & 2 \\
\hline B. plicata & & & & & -- & & & & 2 \\
\hline Crescentilla baltica & & & & & -- & & & & 1 \\
\hline Duplicristatia sp. & & & & -1 & $\ldots .$. & $\ldots$. & $\ldots . .$. & $\ldots .$. & 1 \\
\hline Leperditella prima & & - & & $x$ & & & & & 4 \\
\hline "Paraschmidtella" abnormis & & & & . & $E-$ & -1 & & t & 1 \\
\hline Platybolbina temperata & & $-=$ & - & 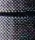 & & & & & 1 \\
\hline Rectella sp. & $\cdots$ & $\cdots \cdots$ & $\cdots$ & & $\cdots \cdots$ & $\cdots \cdots$ & $\ldots$ & $\cdots \cdots$ & 3 \\
\hline Seviculina oanduensis & & & $=$ & f. & - & - & & & 1 \\
\hline Sigmoopsis granulata & & & - & & & & & & 1 \\
\hline Gen. C sp. & $\ldots$ & $\ldots \ldots$ & 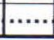 & 4 & $\ldots \ldots$ & $\ldots .$. & $\ldots . .$. & $\ldots$. & 9 \\
\hline
\end{tabular}

Table 3. The stratigraphical distribution of ostracods in the Kalanti boulder based on works by Krause (1892), Sarv (1956, 1959), Neckaja (1966), Sztejn (1985), Sidaravi ciene (1992) and Meidla (unpublished material).

\begin{tabular}{|c|c|c|c|c|c|c|c|c|c|}
\hline Kustavi, Langstet 75/AU/85 & $D_{l}$ & $D_{n}$ & $D_{m}$ & $E$ & $F_{r} a$ & $F_{l} b$ & $F_{1} c$ & $F_{n}$ & No. \\
\hline \multicolumn{10}{|l|}{ OSTRACODES } \\
\hline Bolbina sp. & $\cdots \cdots$ & ..... & .. & & $\cdots$ & $\cdots \cdots$ & $\cdots \cdots$ & ...... & 1 \\
\hline Duplicristatia sp. & & & & & $\ldots$. & $\ldots .$. & $\ldots$. & $\ldots .$. & 1 \\
\hline Hemeaschmidtella sp. & $\cdots \cdots$ & ...... & $\cdots \cdots$ & 8 & $\cdots \cdots$ & $\cdots \cdots$ & $\cdots \cdots$ & $\cdots \cdots$ & 7 \\
\hline Leperditella prima sp. & & -- & --1 & E & & & & & 1 \\
\hline Rectella romboformis sp. & & & ?- & $=$ & -- & -- & -- & -- & 5 \\
\hline Seviculina sp. & & & $\cdots \cdots$ & 8 & $\cdots \cdots$ & $\cdots \cdots$ & $\cdots$ & & 1 \\
\hline Gen. A. sp. & & & --1 & (8) & & & & & 18 \\
\hline Gen. C. sp. & $\cdots \cdots$ & $\cdots \cdots$ & … & .8 & $\cdots \cdot$ & $\cdots$ & $\cdots$ & $\cdots$ & 3 \\
\hline
\end{tabular}

Table 4. The stratigraphical distribution of ostracods in the Kustavi boulder based works by Sarv (1956, 1959), Neckaja (1966) and Meidla (unpublished material).
Both the Kalanti and Kustavi boulders are devoid of acritarchs.

\section{OTHER GROUPS}

Also some other acid-resistant microfossil groups were found that can give further information about faunal associations and the age of samples.

CONODONTS: Some valuable finds were made in the Pyhäranta boulder: among other taxa the zonal species Eoplacognathus robustus Bergström 1971 (Pl. II:18, 19), and also Prioniodus prevariabilis (Bergström 1961) and Scolopodus cornuformis Sergeeva 1963. The range of the former is restricted to the lowermost Uhaku Stage (Männik \& Viira 1990).

INARTICULATE BRACHIOPODS were found in the Pyhäranta boulder: Biernatia cf. holmi Holmer 1989 is known from the Lasnamägi to the Kukruse (or up to Idavere) stages.

FORAMINIFERS: Blastammina sp. group was found in the Pyhämaa boulder, it is widely distributed in the Upper Ordovician (from the Jõhvi to the Porkuni stages). The only finds among the acid-resistant microfossils from the Kalanti and the Kustavi boulder were white foraminifers of genus Psammosphaera, probably $P$. micrograna Eisenack 1932, which is widely distributed in the calcilutites of the Rakvere Stage, but was found also from the Idavere and the Porkuni stages in Estonia.

In addition, unidentified fragments of SCOLECODONTS and MELANOSCLERITES were found only in the Pyhämaa and the Pyhäranta boulders.

\section{DISCUSSION AND CONCLUSIONS}

The present study clearly demonstrates that chitinozoans and acritarchs are valuable for the dating of Ordovician deposits, except reddishbrown Baltic limestone where the only possible 
index fossils are ostracods. However, the best results of dating could be obtained by combining the results from several microfossil groups and well defined lithological description.

In the early Palaeozoic Baltic sections acritarch assemblages alone have very high diversity, although many of species have relatively long vertical distribution. Both acritarchs and chitinozoans are usually well preserved and rather easy to identify. Chitinozoan assemblage is less rich compared to acritarchs, but they are more valuable as guide fossils because of better stratigraphical knowledge in the Baltoscandia. Samples identified were poor in ostracods.

Ostracods are a priori more influenced by facial conditions than chitinozoans and acritarchs (Gailite 1982). However, the knowledge of distribution of ostracods in adjacent areas is good. In the present material ostracods have been the only possible fossils for dating the reddish-brown Baltic limestone (Östersjö limestone), where organic-wall microfossils are destroyed. This type of limestone is not known in the North Estonian investigated sections.

The Pyhäranta boulder originates from the beds of the lower Uhaku according to the data of chitinozoans and conodonts. Acritarchs border the age on the transition of Lasnamägi to Uhaku. Ostracods were too rare for dating.

The Pyhämaa boulder is derived from the beds of late Rakvere stage by all groups investigated.

The Kalanti boulder is by ostracods from the beds of Oandu to Rakvere stages, based on lithology the latter is the most probable.

Also the Kustavi boulder is from the Oandu to Rakvere stages by ostracods. The other groups, except foraminifers were not found.

Both the fossils found and limestone types allow to conclude that the source beds of boulders have been a part of the North Estonian Confacies Belt, although Kalanti and Kustavi boulders differ from the traditional North Estonian lithotypes in some exceed, and at least with respect to the time intervals dated - the Uhaku and Rakvere ages, according to the Pyhäranta and Pyhämaa boulders.

ACKNOWLEDGEMENTS: The chemical analyses were made by $O$. Roos and L. Säga, thin sections by Y. Kestlane (Institute of Geology, Tallinn), X-ray analyses by J. Aruväli (Tartu University). Conodonts were determined by $\mathrm{Dr}$. $V$. Viira (Institute of Geology, Tallinn) and inarticulate brachiopods by Dr. L. Holmer (Uppsala University). Chitinozoans by Ms. $\ddot{U}$. Rosenperk and ostracods by Ms. R. Klaas (Institute of Geology, Tallinn). Figures and tables were drawn by Ms. P. Haikonen (Helsinki University), SCAN photos of chitinozoans and ostracods were taken by Ms. U. Moldov, (Institute of Geology, Tallinn) and photos of samples by Mr. J. Väätäinen (Geological Survey of Finland, Espoo). Grateful thanks are expressed to all these people. 


\section{REFERENCES}

Eisenack, A., 1965. Die Mikrofauna der Ostseekalke. 1. Chitinozoen, Hystrichosphären. Neues Jahrbuch für Geolologische und Paläontologische Abhandlung 123:2, 115-148.

Eisenack, A., 1972. Beiträge zur Chitinozoen-Forschung. Paleontographica A, 140:4-6, 117-130.

Eisenack, A., 1976. Mikrofossilien aus dem Vaginatenkalk von Hälludden, Öland. Palaeontographica A, 154:4-6, 181-203.

Evitt, W.R., 1963. A discussion and proposals concerning fossil dinoflagellates, hystrichospheres and acritarchs. Proceedings Natn. Acad. Sci. 49, 158-164.

Gailite, L.K., 1982. Ostracods. In: Ulst, R.Zh., Gailite, L.K. \& Jakoleva, V.I. (eds). Orodvician of Latvia, 114132. (in Russian)

Grahn, Y., 1980. Early Ordovician Chitinozoa from Öland. Sveriges Geologiska Undersökning, Serie C, 775, 41 p.

Grahn, Y., 1981a. Middle Ordovician Chitinozoa from Öland. Sveriges Geologiska Undersökning, Serie C, $784,51 \mathrm{p}$.

Grahn, Y., 1981b. Ordovician Chitinozoa from the Stora Asbotorp boring in Västergötland, south-central Sweden. Sveriges Geologiska Undersökning, Serie C, 787, $40 \mathrm{p}$.

Grahn, Y., 1982. Caradocian and Ashgillian Chitinozoa from the subsurface of Gotland. Sveriges Geologiska Undersökning, Serie C, 788, 66 p.

Grahn, Y., 1984. Ordovician Chitinozoa from Tallinn, northern Estonia. Review of Paleobotany and Palynology 43, 5-31.

Jaanusson, V., 1972. Constituent analysis of an Ordovician limestone from Sweden. Lethaia 5, 217-237.

Jaanusson, V., 1976. Faunal dynamics in the Middle Ordovician (Viruan) of Balto-Scandia. In: Basset, M.G. (ed.), The Ordovician System. Proc. Palaeontological Association symposium 1974, Birmingham, Univ. Wales Press and National Museum of Wales, Cardiff, 301-326.

Kjellström, G., 1971. Middle Ordovician microplankton from the Grötlingbo Borehole No. 1 in Gotland, Sweden. Sveriges Geologiska Undersökning, Serie C, No. 669, Årsbok 65:15, 35 p.

Krause, A., 1892. Neue Ostracods aus märkischen Silurgeschieben. Z. Deutschland Geologische Ges. 44, 383-399.

Laufeld, S., 1967. Caradocian Chitinozoa from Dalarna, Sweden. Geologiska Förenningen i Stockholm Förhandlingar 89:3, 275-349.

Lundin, R.F., 1988. Is Neckajatia an ancestor of the platycope ostracods? In: Hanai, T., Ikeya, N., Ishizaki,
K. (eds). Evolutionary biology of Ostracods, its fundamentals and applications. Proc. Ninth Int. Symp. on Ostracoda, Shizuoka, Japan, 29 July - 2 August 1985. Developments in palaeontology and stratigraphy 11 , 1051-1060.

Martinsson, A., 1956. Neue Funde kambrischer Gänge und ordovischer Geschiebe im südwestlichen Finnland. Bulletin of Geological Institute of University in Uppsala XXXVI, 79-105.

Männik, P. \& Viira, V., 1990. Conodonts. In: Kaljo, D. \& Nestor, H. (eds). Field Meeting Estonia 1990. An Excursion Guidebook. Institute of Geology, Estonian Academy of Sciences, 84-89.

Männil, R., 1986. Stratigraphy of the kukersite-bearing deposits. In: Puura, V. (ed.). Geology of the kukersitebearing beds of the Baltic oil shale basin. Institute of Geology, Estonian Academy of Sciences, Tallinn 1224. (in Russian).

Meidla, T., 1986. Neue Ostracoden aus dem Ordovizium des Baltikums. Proceedings of the Academy of Sciences of the Estonian S.S.R. Geology 35:1, 10-19. (in Russian with German summary)

Meidla, T., 1993. The ostracod genus Longiscula Neckaja 1958 in the Ordovician of the East Baltic. Neues Jahrbuch für Geolologische und Paläontologische Abhandlung 188:3, 289-303.

Meidla, T. (in press). Upper Ordovician ostracods of Estonia.

Neckaja, A.I., 1966. Ordovician and Silurian ostracods of the U.S.S.R. (the families Schmidtellidae, Rectellidae, Longisculidae and some representatives of other families). Proc. All-Union Sci.-Res. Geol. Prosp. Inst. (VNIGRI) 251, 1-76 (in Russian).

Nölvak, J. \& Grahn, Y., 1993. Ordovician chitinozoan zones from Baltoscandia. Review of Paleobotany and Palynology 79, 245-269.

Sarjeant, W.A.S. \& Stancliffe, R.P.W., 1994. The Micrhystridium and Veryhachium complexes (Acritarcha: Acanthomorphitae and Polygonomorphitae): a taxonomic reconsideration. Micropalaeontology 40:1, 1-77.

Sarv, L., 1956. New ostracod species from the Vasalemma Stage (Upper Ordovician of the Estonian S.S.R). Proceedings in Institute of Geology, Estonian Academy of Sciences, Tallinn, Estonian S.S.R. 1, 30-40. (in Russian)

Sarv, L., 1959. The Ordovician ostracods of the Estonian S.S.R., Proc. Institute of Geology, Estonian Academy of Sciences, Tallinn, Estonian S.S.R. 4, 1-206. (in Russian)

Schallreuter, R.E.L., 1978. On Duplicristatia asymmetrica gen. et sp. n. In: A Stereo-Atlas of Ostracod Shells 5 (7), 49-56. 
Schulz, G., 1967. Mikrofossilien des oberen Llandovery von Dalaerne (Schweden). Sonderveröff. Geologische Institut Universität Köln 13, 175-187.

Sidaravičiene, N., 1992. The Ordovician ostracods of Lithuania. Vilnius, 1-252. (in Russian).

Sohn, I.G., 1961. Techniques for preparation and study of fossil ostracods. In: Moore, R.C. (ed.). Treatise on invertebrate paleontology, Q Arthropods 3, 64-70. Geological Society of Amererica \& University Kansas Press.

Sztejn, J., 1985. Malzoraczki ordowiku w PlnocnoWshodnieji Polsce. Biuletin Instut Geology 350, 53-89.

Sztejn, J., 1989. Stratigrafia mikropaleontologiczna ordowiku Lubelszczyzny i zachodniej Czeci obnizenia Podlaskiego. Biuletin Instut Geology 363, 69-92.

Tynni, R., 1975. Ordovician hystrichopheres and chitinozoans in limestone from the Bothnian Sea. Geological Survey of Finland, Bulletin 279, 59 p.

Ulrich, E.O., 1894. The Lower Silurian Ostracoda of Min- nesota. Minnesota Geol. and Nat. Hist. Surv., Rep. 3("), Minneapolis.

Uutela, A., 1989. Age and provenance of sedimentary erratics on the coast of southwestern Finland. Geological Survey of Finland, Bulletin 349, 100 p.

Uutela, A. \& Tynni, R., 1991. Ordovician acritarchs from the Rapla borehole, Estonia. Geological Survey of Finland, Builetin 353, $135 \mathrm{p}$.

Vannier, J.M.C., Siveter, D.J. \& Schallreuter, R.E.L., 1989. The composition and palaeogeographical significance of the Ordovician ostracod faunas of southern Britain, Baltoscandia and Ibero-Armorica. Palaeontology 32:1, 163-222.

Vidal, G., 1988. A palynological preparation method. Palynology 12, 215-220.

Winterhalter, B., Flóden, T., Ignatius, H., Axberg, S. \& Niemistö, L., 1981. Geology of the Baltic Sea. In: Voipio, A. (ed.), The Baltic Sea. Elsevier Oceanography Series 30, 1-121. 


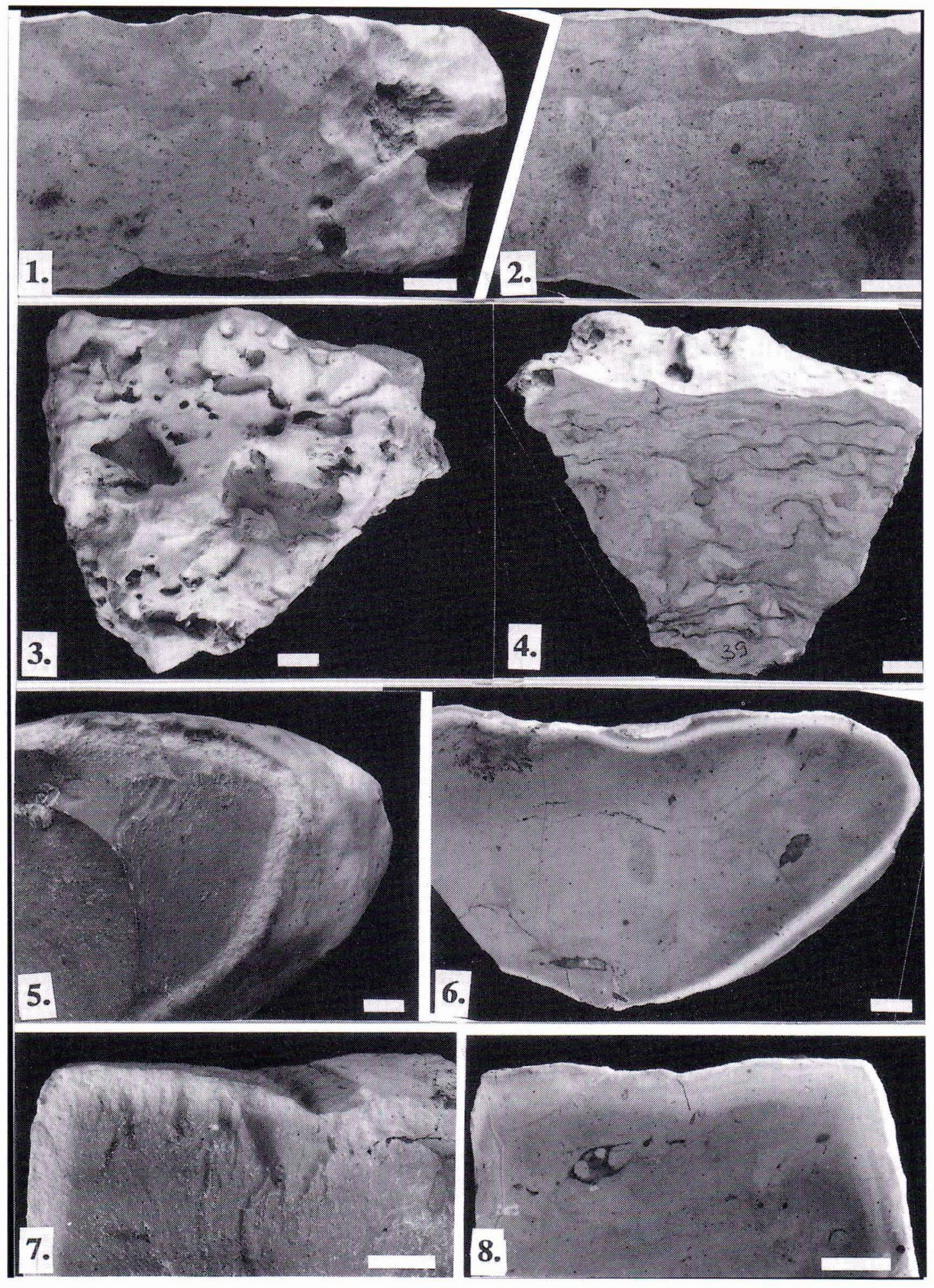




\section{Plate I.}

Erratic boulders from south-western coast of Finland. 1,2: Pyhäranta, grey calcarenite. 3, 4:

Pyhämaa, yellow-grey calcilutite. 5, 6: Kalanti, reddish-brown calcilutite. 7, 8: Kustavi, reddishbrown calcilutite. Photos by J. Väätäinen, Geological Survey of Finland, Espoo.

Bar equals $1 \mathrm{~cm}$. 


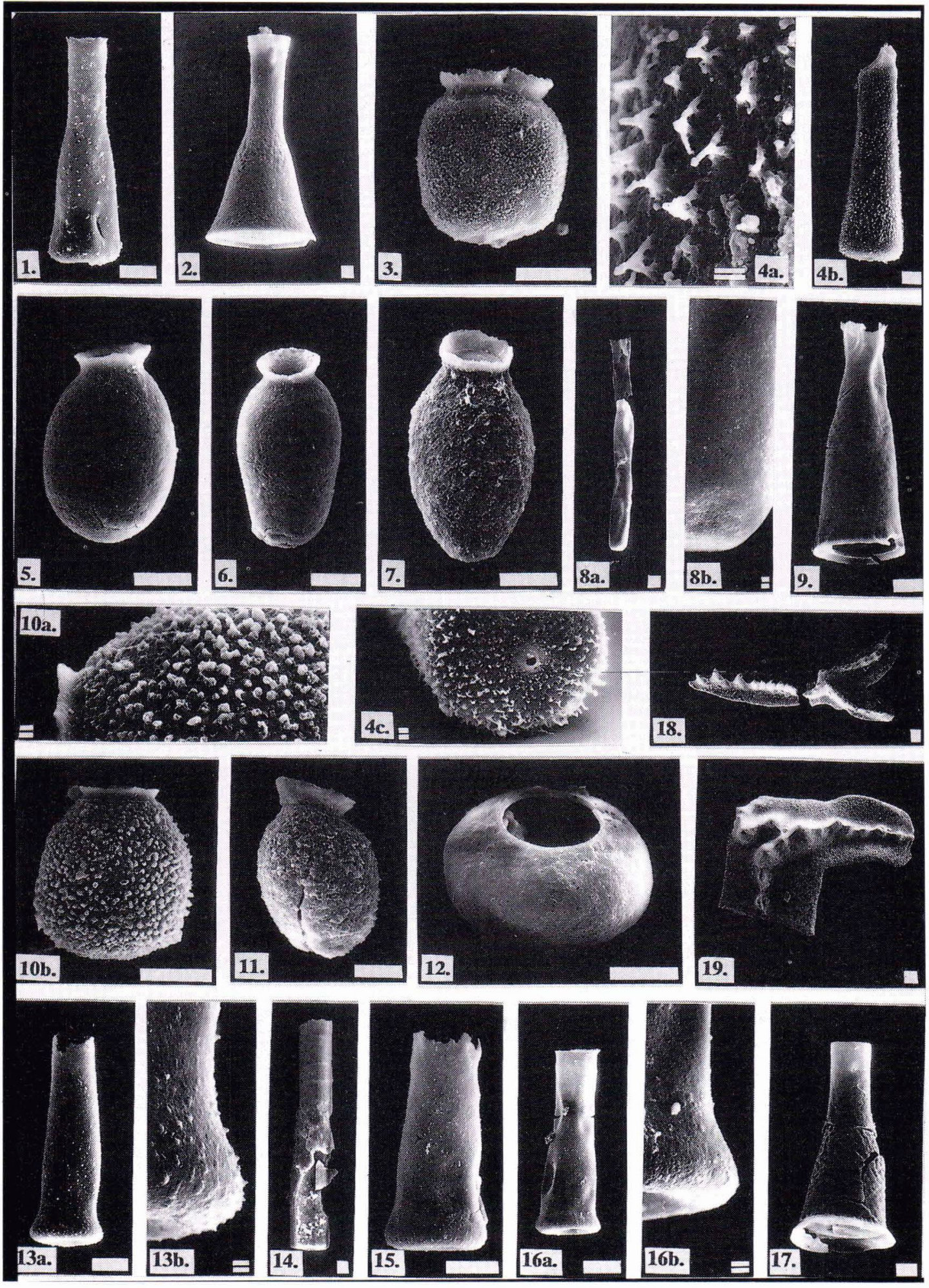




\section{Plate II}

Chitinozoans (Ch) and conodonts (Co) from the Pyhäranta and Pyhämaa boulders.

1. Belonechitina wesenbergensis brevis (Eisenack 1965). Ch 1528; Pyhämaa.

2. Cyathochitina campanulaeformis (Eisenack 1931). Ch 1530; Pyhämaa.

3. Desmochitina minor Eisenack 1931. Ch 1526; Pyhämaa. 4. Belonechitina robusta (Eisenack 1959). Ch 1527; Pyhämaa. 4a. Ornamentation, spines with multiramose roots coalescent. $4 \mathrm{~b}$.

Almost complete specimen with broken aperture. $4 \mathrm{c}$. Base.

5. Desmochitina ovulum Eisenack 1962. Ch 1511; Pyhäranta.

6. Desmochitina elongata Eisenack 1958. Ch 1524; Pyhäranta. 7. Desmochitina elongata

Eisenack 1958. Ch 1522; Pyhäranta. 8a. Rhabdochitina gracilis Eisenack 1962. Ch 1514;

Pyhämaa. Broken, almost complete specimen. 8b. Balsal edge.

9. Cyathochitina calix (Eisenack 1931). Ch 1510; Pyhäranta. Almost complete specimen with broken base.

10a. Ornamentation of Desmochitina erinacea. 10b. Desmochitina erinacea Eisenack 1962. Ch 1512; Pyhäranta.

11. Desmochitina amphorea Eisenack 1931. Ch 1516; Pyhäranta. 12. Desmochitina complinata Eisenack 1932. Ch 1513; Pyhäranta. Almost complete specimen with broken collar.

13a. Belonechitina micracantha (Eisenack 1931). Ch 1521; Pyhäranta. Almost complete specimen with broken aperture. 13b. Ornamentation: small simple spines.

14. Conochitina clavaherculi Eisenack 1959. Ch 1517; Pyhäranta.

15. Conochitina primitiva Eisenack 1939. Ch 1520; Pyhäranta. 16. Conochitina primitiva

Eisenack 1939. Ch 1518; Pyhäranta. 17. Cyathochitina kuckersiana (Eisenack 1934). Ckh

1523; Pyhäranta.

18. Eoplacognathus robustus Bergström 1971. Co 0028; Pyhäranta.

19. Eoplacognathus robustus Bergström 1971. Co 0035; Pyhäranta.

Bar equals $100 \mu \mathrm{m}$. 


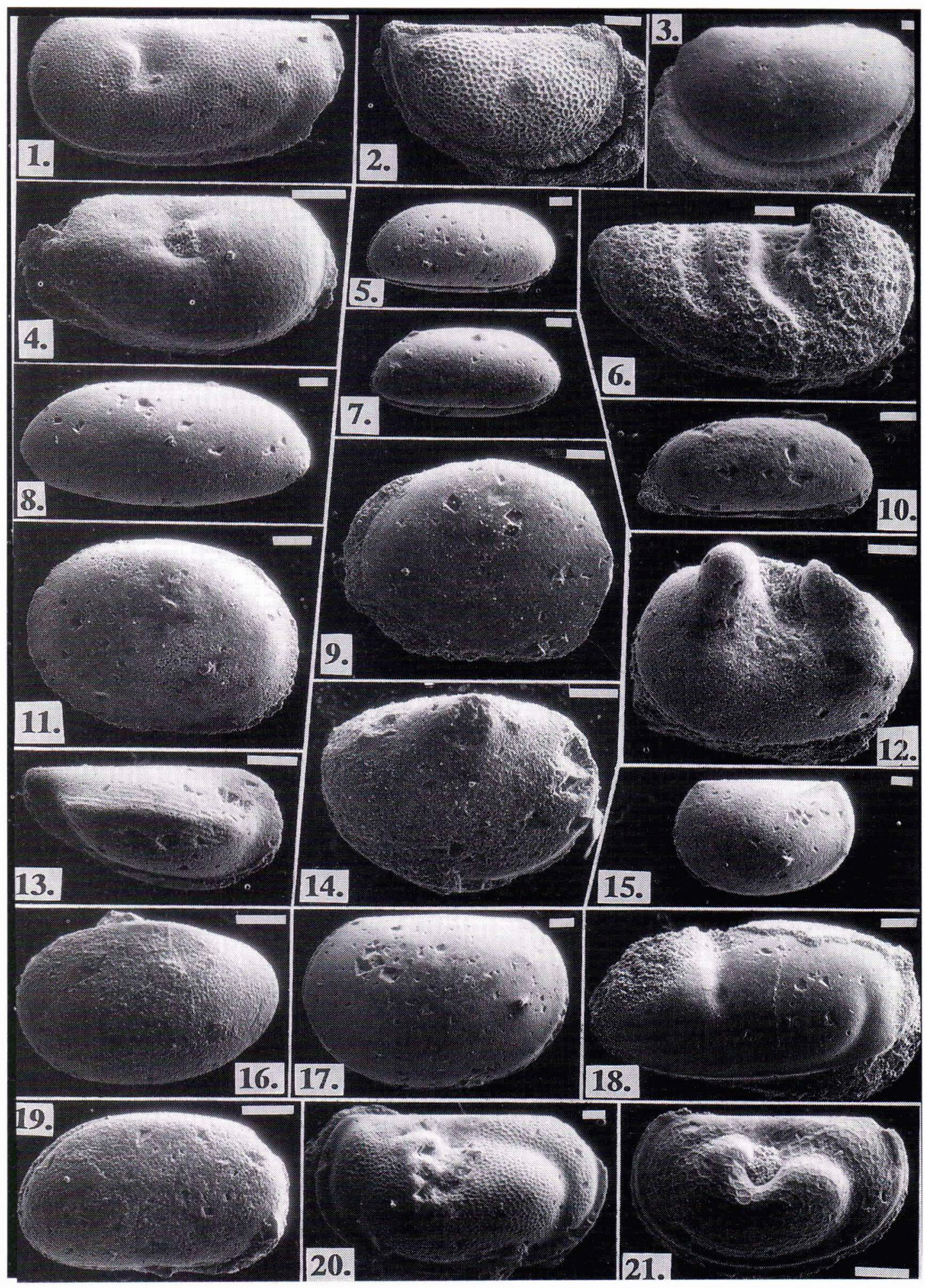




\section{Plate III}

Ostracods (Os) from the Kalanti, Kustavi and Pyhämaa boulders.

1. Seviculina oanduensis (Sarv 1956). Carapace Os 3254, left view; Kalanti.

2. Platybolbina (Reticulobolbina) temperata Sarv 1959. Juvenile, right valve. Os 3252, lateral view; Kalanti.

3. Platybolbina (Platybolbina) orbiculata Sarv 1959. Left? incomplete valve. Os 3251 , lateral view; Pyhämaa.

4. Seviculina sp. Incomplete carapace. Os 3255 , right view; Kustavi.

5. Gen. A sp. n. Juvenile carapace. Os 3274 , right view; Kustavi.

6. Sigmoopsis granulata Sarv 1956. Right valve. Os 3253, lateral view; Kalanti.

7. Gen. $A$ sp. n. Juvenile carapace. Os 3276 , right view; Kustavi.

8. Gen. A sp. n. Juvenile carapace. Os 3273, right view; Kustavi.

9. "Paraschmidtella" abnormis (Sideravičiene 1975). Damaged carapace. Os 3262, left view, L-0.64; H-0.30; Kalanti.

10. Longiscula sp. Deformed carapace. Os 3271, right view; Pyhämaa.

11. "Paraschmidtella" abnormis (Sideravičiene 1975). Carapace. Os 3263, right view; Kalanti.

12. Crescentilla baltica Neckaja 1966 . Incomplete right? valve. Os 3260 , lateral view, L-0.52;

H-0.25; Kalanti.

13. Duplicristatia sp. Carapace. Os 3270, right view; Kustavi.

14. Hemiaechminoides minusculus Meidla 1986. Carapace. Os 3267, left view; Pyhämaa.

15. Leperditella prima Sarv 1956. Juvenile carapace. Os 3269, right view; Kalanti.

16. Gen. B. sp. n. Juvenile left valve. Os 3279 , lateral view, L-0.52; H-0.33; Pyhämaa.

17. Leperditella prima Sarv 1956. Preadult carapace. Os 3268, right view; Kustavi.

18. Bolbina plicata (Krause 1892). Incomplete left valve. Os 3258, lateral view; Kalanti.

19. Neckajatia? sp. Carapace. Os 3278 , left view; Pyhämaa.

20. Bolbina major (Krause 1892). Tecnomorphe carapace. Os 3257, left view; Pyhämaa.

21. Bolbina major (Krause 1892). Juvenile carapace. Os 3256, left view; Kalanti.

Bar equals $100 \mu \mathrm{m}$. 


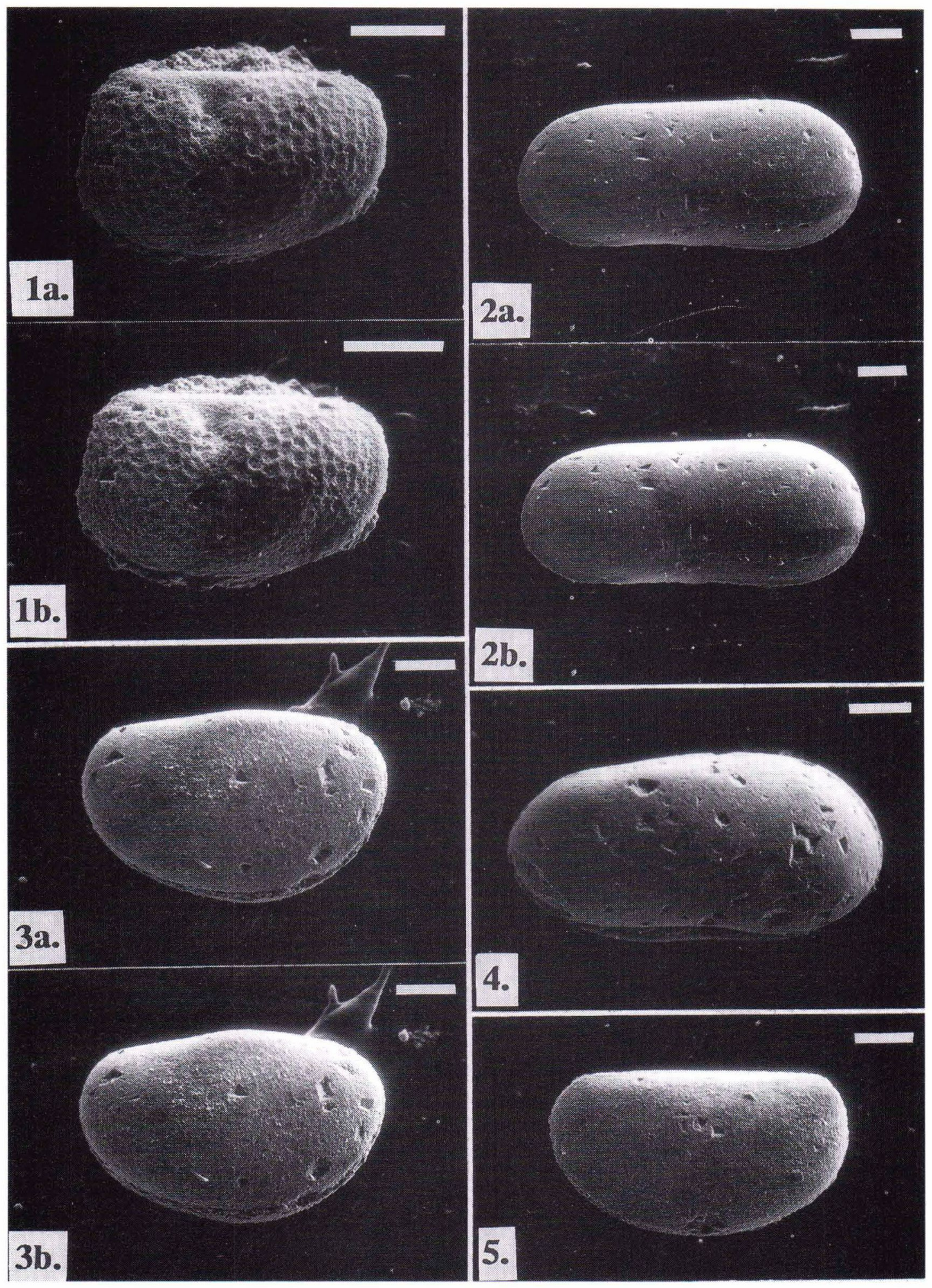




\section{Plate IV}

Ostracods (Os) from the Kalanti, Kustavi and Pyhämaa boulders.

1a, b. Bolbina sp. Incomplete carapace. Os 3259, left view (stereo-pair), L (incomplete)-0.33;

H- 0.20 ; Kustavi.

2a, b. Gen. C. sp. n. Carapace. Os 3280, left view (stereo-pair); Kalanti.

3a, b. Hemeaschmidtella sp. Carapace. Os 3266, left view (stereo-pair), L-0.76; H-0.38; Kustavi.

4. Rectella romboformis Neckaja 1966. Carapace. Os 3272, right view; Kustavi.

5. Hemeaschmidtella sp. Carapace. Os 3265, left view; Kustavi.

Bar equals $100 \mu \mathrm{m}$. 


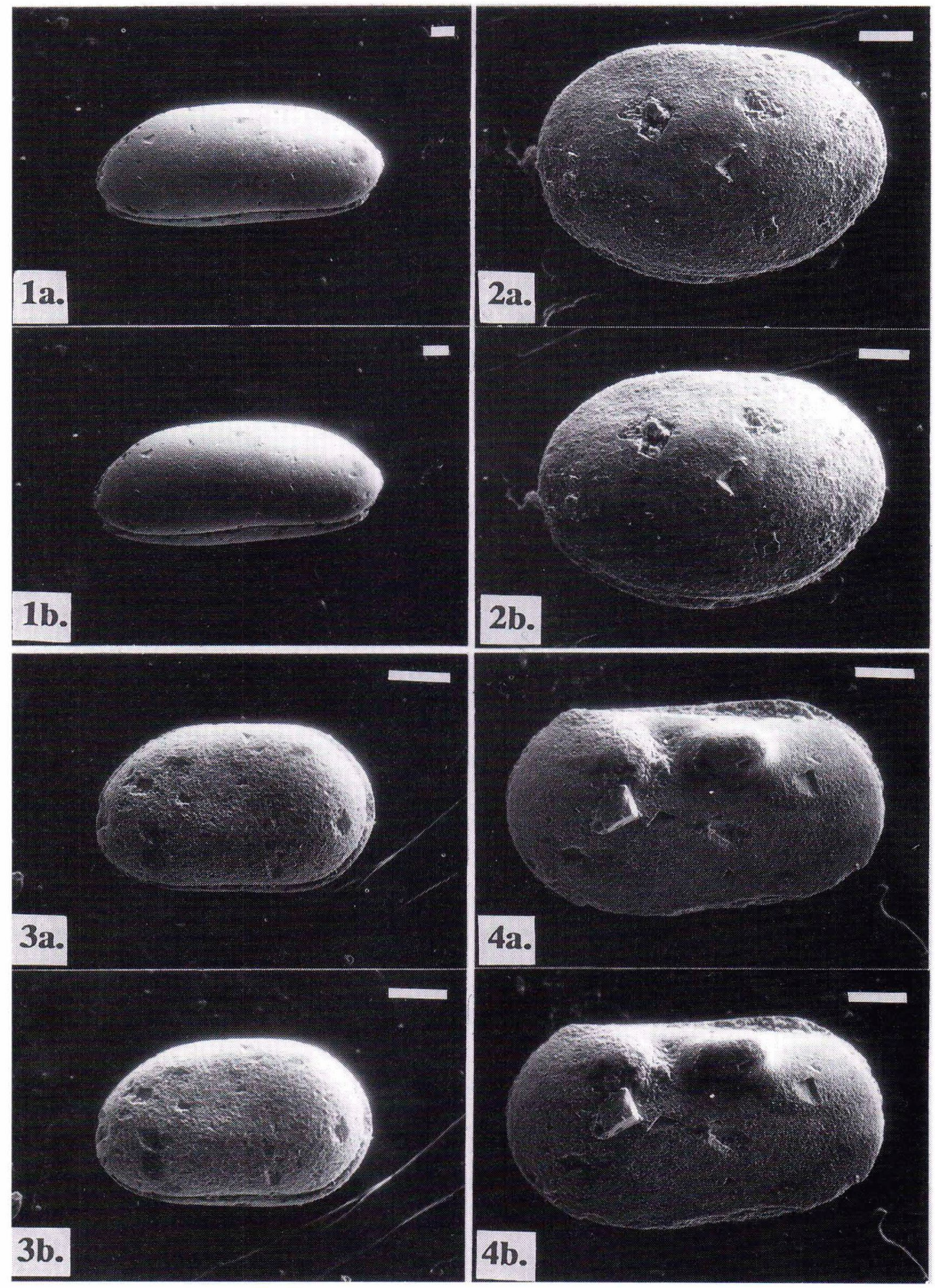




\section{Plate V}

Ostracods (Os) from the Kalanti, Kustavi and Pyhämaa boulders.

1a, b. Gen. A. sp. n. Carapace. Os 3275, right view (stereo-pair); Kustavi.

2a, b. Baltonotella? sp. Carapace. Os 3264, left view; Pyhäranta.

3a, b. Neckajatia? sp. n. Carapace. Os 3277, right view (stereo-pair); Pyhämaa.

4a, b. Pseudulrichia inarguta (Neckaja 1966). Carapace. Os 3261, left view (stereo-pair); Pyhämaa.

Bar equals $100 \mu \mathrm{m}$. 


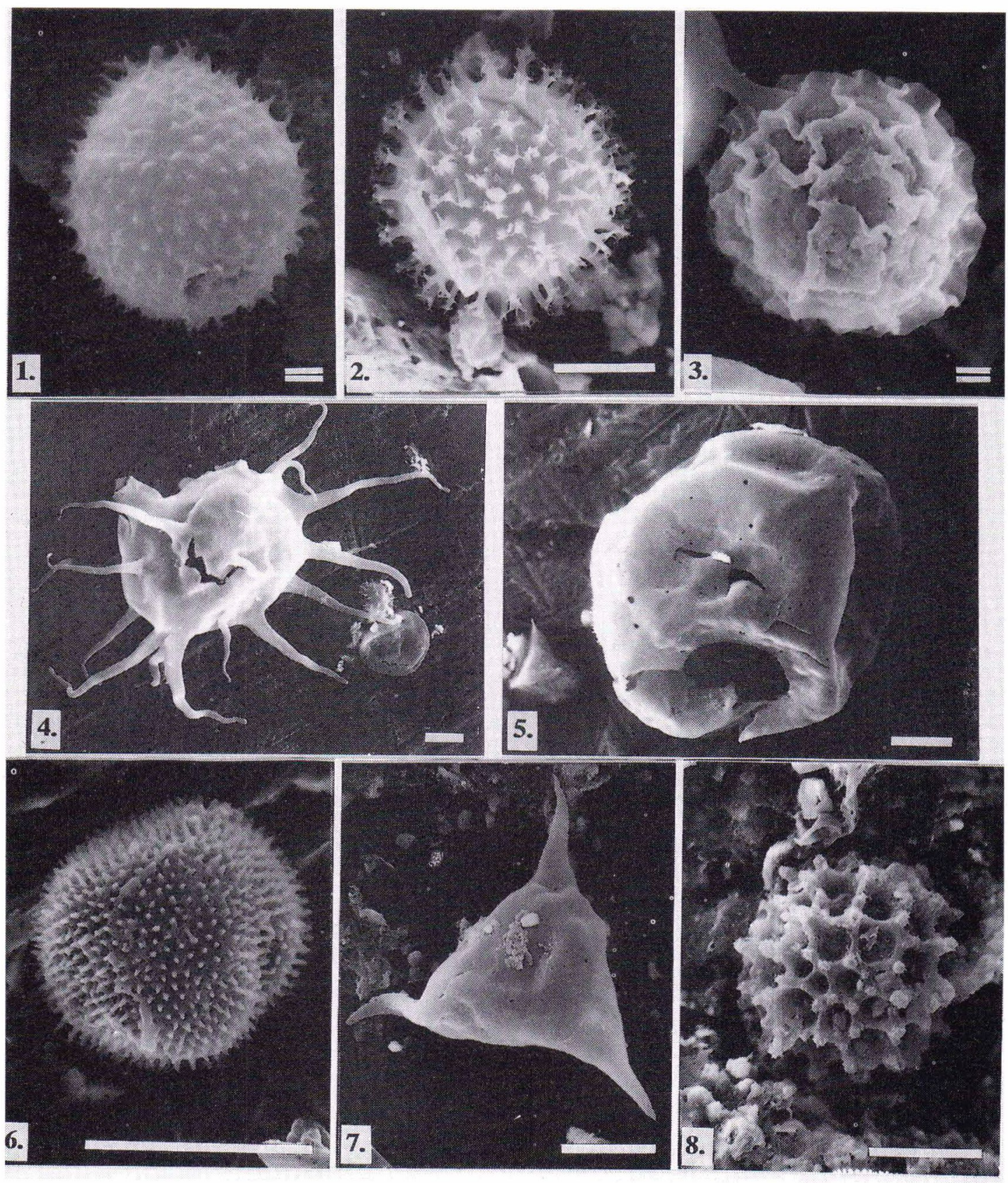




\section{Plate VI}

Acritarchs from the Pyhäranta and Pyhämaa boulders.

1. Revinotesta granulosa Uutela \& Tynni 1991; Pyhämaa.

2. Tunisphaeridium spinosissimum Uutela \& Tynni 1991; Pyhämaa.

3. Cymatiosphaera crispa Uutela \& Tynni 1991; Pyhämaa.

4. Baltisphaeridium mochtiensis Gorka 1969; Pyhäranta.

5. Tranvikium polygonale Tynni 1982; Pyhäranta.

6. Filisphaeridium eatonense (Downie 1959) Sarjeant \& Stancliffe 1994; Pyhäranta.

7. Veryhachium reductum (Deunff 1959) Downie \& Sarjeant 1965; Pyhäranta.

8. Timofeevia nodosa Uutela \& Tynni 1991; Pyhäranta.

Bar equals $10 \mu \mathrm{m}$ and doublebar $1 \mu \mathrm{m}$.

\section{APPENDIX}

Description of ostracods:

Genus A. sp. n.

Pl. III:5, 7, 8; Pl. V:1a, b.

Description: Elongate to oval, slightly trapezoidal podocope, characterizes a left-over-right overlap along the entire free margin and by a distinct overreach of the left valve of the ventral margin. The latter feature and the character of convexity (the greatest convexity occurs in the ventral part of the carapace, particularly by the left valve) make a clear difference between the current species and metacope genera common in the East Baltic, like Rectella or Longiscula. In these features the new species is similar to Krausella in Ulrich (1894), but lacks a posteroventral process on the right valve.

Genus B. sp. n.

Pl. III:16.

Description: A small (L- 0.52; H - 0.33) left valve of a metacope is strongly convex and with high dorsal umbo. It is probably conspecific to the material found in Estonia and tentatively referred to Daleiella.

Baltonotella? sp.

Pl. V:2a, b.

Description: A small, amplete, moderately convex carapace has the greatest width in the central area. Hinge line is short $(<0.5$ of the total length), without distinct dorsum and a slight left-over-right overreach occurs the hinge margin. Right valve slightly overlaps the left one probably along the entire free margin (the marginal part of the right valve is damaged posteroventrally). Surface is smooth.

Baltonotella and Brevidorsa differ from each other by lacking or having the striated admarginal sculpture, accordingly. Current single specimen from Pyhäranta boulder possesses no definite admarginal sculpture, but a very indefinite pattern can be recognized posteroventrally. Both genera are distributed throughout the Middle and Upper Ordovician in the East Baltic, Baltonotella has also been referred to the Lower Ordovician.

Bolbina sp.

Pl. IV:1a, b.

Description: A very small, moderately convex carapace has anterior end damaged (L. (incomplete)- $0.33 ; \mathrm{H}-0.20$ ). Dorsaly situated sulcus is short, semisulcus in the posteroventral region of the valve is poorly developed. Surface is coarsely reticulated.

The identification of a juvenile specimen in the Kustavi boulder is difficult, the specimen may belong to a known species Bolbina major.

Genus $C$. n. sp.

Pl. IV:2a, b.

Description: The species is small ( $\mathrm{L}-0.76$; $\mathrm{H}-0.38$ ) elongate, with straight dorsal and concave ventral margins, 
the anterior end is more narrowly rounded than the posterior one. The carapace is moderately convex and the free margin is slightly flattened along the anteroventral, ventral and posteroventral borders, except the anterior part of the ventral margin which is characterized by a slight left-overright overlap. The surface is smooth. The figured specimen is probably a juventile one, but larger ones have been damaged. Similar forms occur in Estonia but their variation is not studied in detail. Due to the obvious heterogenity of this group its stratigraphic range cannot be estimated correctly.

Duplicristatia sp.

Pl. III:13.

Description: A small specimen from Kalanti boulder (L - 0.52; H - 0.25) differs from Duplicristatia asymmetrica in Schallreuter (1978) by having a minor ventral crista on the valve surface. Probably the same form has been described by Sidaravičiene (1992, Pl. 52:810) as Duplicristatia sp. A from the Rakvere Stage in Lithuania. In Estonia Duplicristatia specimens occur in the interval from the Rakvere Stage up to the topmost Ordovician.

\section{Hemeaschmidtella sp.}

Pl. IV:3a, b, 5 .

The dorsal umbo on the right valve of elongate Hemeaschmidtella is poorly developed. This material is similar in outline to Hemeaschmidtella? sp. A by Sidaravi iene $(1992$, Pl. 46:8, 9) but possesses the free margin structure typical for Hemeaschmidtella. Conspecific specimens are recognized from the Oandu to Nabala stages in Estonia. They may belong to Hemeaschmidtella ovata Sztejn (Sztejn 1989). The genus is characteristic of the Middle and Upper Ordovician.

Longiscula sp.

Pl. III: 10 .

Description: A small ( $\mathrm{L}-0.64 ; \mathrm{H}-0.30$ ), elongate to oval, moderately convex, slightly deformed carapace has an arched dorsal margin, continuously going over at both ends, which are narrowly rounded near the vertical margin. Ventral margin is slightly concave in the middle. Listed features allow to assign the current specimen to Longiscula without species identification.

Neckajatia? sp.

Pl. III:19; Pl. V:3a, b.

Description: The taxonomic position of this small, slightly asymmetrical nonpalaeocope ostracod is unclear due to the difficulties of the orientation. The adductor muscle spot surrounded by a groove is distinct, and is located more close to the narrowly rounded end of the carapace ( $\mathrm{Pl}$. V: $3 \mathrm{a}, \mathrm{b})$. If this end should be treated as the anterior one, a right-over-left overlap must be considered, which is untypical of the metacopes. By these conditions the species could be assigned to Neckajatia Schallreuter 1974, early representatives of which have been treated by Lundin (1988) as a link between leperditellaceans and first true platycopes. Anyway, the taxonomic position to the genus Neckajatia will be the oldest known representative of the genus.

In the Estonian collection such forms have been recorded mainly in the Rakvere and Nabala stages, questionably also in the Oandu and Vormsi stages (Meidla in prep.).

\section{Seviculina sp.}

Pl. III:4.

An incomplete specimen from Kustavi boulder may be conspecific with S. reticulata Meidla 1986. The latter occurs in Estonia in the Rakvere and Nabala stages, but rare specimens of Seviculina (probably another species) are known in the Nabala and Vormsi stages also. The first representatives of the genus appear in the Oandu Stage in the East Baltic. 